\title{
BIÓPSIA ESTEREOTÁXICA GUIADA POR IMAGEM NAS LESÕES DO SISTEMA NERVOSO CENTRAL
}

\author{
JOSÉ AUGUSTO NASSER*, CARLOS IVAM CONFORT**, \\ ANDREI FERRAZ**, JOSÉ CARLOS ESPERANÇA***, FRANCISCO DUARTE****
}

\begin{abstract}
RESUMO - Em 44 biópsias estereotáxicas, guiadas por imagem, no período de agosto-1995 até março-1997, as lesões encontradas foram (ordem de frequência): gliomas (glioblastoma multiforme o mais comum), linfoma primário e demais lesões ligadas à síndrome da imunodeficiência adquirida, metástases, vasculites, cisto aracnóideo, doença de Creutzfeldt-Jakob, nódulo fibroglial calcificado. A idade variou de 1 a 83 anos. 41 lesões eram supratentoriais, 2 infratentoriais e uma da calota craniana. A mortalidade foi nula e a morbidade 2,3\%. A literatura é revista. $\mathrm{O}$ autores concluem que este procedimento é seguro e eficaz.
\end{abstract}

PALAVRAS-CHAVE: biópsia estereotáxica, glioma, metástase cerebral, linfoma, neuroinfecção.

\section{Image guided stereotactic biopsy of central nervous system lesions}

ABSTRACT - In a series of 44 image guided stereotactic biopsy from August 1995 until March 1997, findings were as follows (frequency order). Tumors, glioblastoma was the most frequent. Primary lymphoma and other conditions associated to AIDS. Metastasis, three cases. Vasculites, two cases. Arachnoid cyst, Creutzfeldt-Jakob, cortical degeneration, inespecific calcification (one case each). The age varied from 1 to 83 years. Forty one lesions were supratentorial, two infratentorial, and one was outside the brain (dura and skull) and we used stereotaxy to localize it. There was no mortality and morbidity was $2.3 \%$. The literature is reviewed. We conclude that this procedure is safe and highly diagnostic.

KEY WORDS: stereotactic brain biopsy, glioma, brain metastasis, lymphoma, CNS infections.

A biópsia estereotáxica (BE), de lesões encefálicas ou cranianas, guiada por imagem, vem sendo hoje parte de um arsenal diagnóstico que qualquer centro especializado em neurociências deve possuir. Todos os recursos em informática, todas as modernizações dos equipamentos disponíveis no mercado em estereotaxia vieram trazer a este procedimento segurança e qualidade. Hoje faz parte dos procedimentos chamados minimamente invasivos, podendo ser realizado em pacientes com riscos maiores, mais idosos, imunossuprimidos e que necessitam de diagnóstico para nortear a terapêutica, como os portadores da AIDS. Este método evita que muitos pacientes sejam submetidos a cirurgias maiores desnecessárias, outras vezes orientam um planejamento cirúrgico adequado ou, como nos abscessos e cistos, pode ser o tratamento ou parte importante dele. Com estes objetivos nós procuraremos relatar nossa experiência em BE, nossos acertos e nossas dificuldades. Definimos hoje a equipe de biópsia como um tripé formado por: neuro-radiologista, neurocirurgião e neuropatologista. A integração perfeita desta equipe maximiza a positividade do procedimento.

* Mestrando em Neurocirugia (UNIFESP - Escola Paulista de Medicina, São Paulo), Neurolife Laboratórios, Estereolife Neurocirurgia Funcional e Estereotáxica (NLL/ELNFE); ** NLL/ELNFE e Hospital Universitário Clementino Fraga Filho da Faculdade de Medicina da Universidade Federal do Rio de Janeiro (UFRJ);***Professor Adjunto da Anatomia Patológica da FM/UFRJ; ****Professor Titular de Anatomia Patológica da FM/UFRJ, Pesquisador do CNPq. Aceite: 12-janeiro-1998.

Dr. José Augusto Nasser - Rua do Russel 450/802 - 22210-010 Rio de Janeiro RJ - Brasil. 


\section{MÉTODO}

\section{Material clínico}

No período de agosto-1995 a março-1997, 44 pacientes foram selecionados para a BE. Todos foram indicados inicialmente pelos seus próprios médicos e encaminhados com seus exames de imagem ao nosso Serviço. Os pacientes selecionados apresentavam em suas lesões algumas destas características aos exames de imagem: a) profunda, b) múltipla, c) local eloquente, d) pequena. Uma necessidade de esclarecimento histológico, visando estabelecer uma abordagem adequada clínica ou cirúrgica, ou um risco cirúrgico elevado para procedimentos maiores, também determinaram a indicação da BE .

No caso dos portadores do vírus HIV, todos foram submetidos ao escalonamento diagnóstico e terapêutico descrito por Levy ${ }^{1,2}$ que estabelecia o momento correto do paciente ser biopsiado. Estes pacientes neurologicamente sintomáticos foram submetidos a estudos de imagem: a) tomografia computadorizada (TC) com contraste, em alguns casos o duplo contraste foi necessário para melhor visualização dos limites da lesão; b) ressonância magnética (RM) com contraste (gadolíneo). Se os estudos fossem normais ou mostrassem apenas atrofia, o exame de líquor era realizado; caso este fosse normal, o paciente era orientado a retornar ao serviço mensalmente. Quando se observassem lesões múltiplas, um teste terapêutico para toxoplasmose era iniciado e monitorado por duas semanas com imagem, se houvesse regressão das lesões o tratamento seria intensificado, caso contrário a BE estaria indicada. Para as lesões solitárias e para pacientes instáveis neurologicamente, a BE era indicada prontamente.

Alguns pacientes com lesão cortical superficial, ou mesmo diplóica tomando a dura máter, ou atrofia cortical mostrada na RM localizada, foram mapeados por estereotaxia e biopsiados, sendo incluídos nesta série.

\section{Procedimento cirúrgico}

Em todos os casos utilizou-se o Sistema Esterotáxico ETM-03B (Micromar, Diadema SP) e o programa msa.out para o cálculo das coordenadas (Armando Alaminos Bouza). Para o procedimento cirúrgico o paciente é internado no mesmo dia. O halo é fixado sob anestesia local (lidocaína a 2\%). Imediatamente após, o paciente é levado para o centro de imagens onde realizamos a estereotomografia do crânio com contraste venoso. Todas as informações são enviadas à central de trabalho (Epson- 4SLC) que, através do programa msa.out, nos permite obter todas as coordenadas estereotáxicas dos alvos eloquentes pelos exames de imagem. O paciente é então levado ao centro cirúrgico. Sob sedação (propofol) e anestesia local, usando novamente lidocaína a $2 \%$, é feita a incisão com os dados fornecidos pelo programa e aplicados ao sistema estereotáxico. A trepanação é orientada pelo sistema estereotáxico, assim como a abertura dural. A hemostasia cautelosa, com auxílio de lupa, é rotina no intuito de evitar complicações. Após todo o sistema revisto, uma pinça de biópsia tipo janela lateral (Sedan) ${ }^{3}$ ou sacabocados (Gildemberg) ${ }^{4}$ é introduzida e os fragmentos são removidos, para que o material seja examinado pelo neuropatologista durante a intervenção. Nos casos de tumores, uma biópsia seriada é realizada. Algumas vezes, o diagnóstico já pode ser confirmado em sala, outras vezes apenas a positividade do material é evidenciada e o diagnóstico definitivo é estabelecido em preparação com parafina, posteriormente. De qualquer forma, todas as amostras são exaustivamente analisadas e submetidas à bateria de preparações disponíveis (imunohistoquímicas). Os pacientes habitualmente permanecem no hospital por 24 horas após o procedimento.

\section{Histopatologia}

O material recebido a fresco durante o ato cirúrgico é colocado sobre superfície de vidro; filiforme, medindo cerca de $4 \mathrm{~mm}$ e é estirado, retirando-se mínimos fragmentos das duas pontas, dos quais serão confeccionados esfregaços com auxílio de lâmina de bisturí. Estes são imersos imediatamente em etanol absoluto por um minuto. A parte restante do material retirado é colocada em solução fixadora de formol a 10\%. Então, as lâminas já fixadas, são hidratadas em etanol em concentrações decrescentes (álcool a 90\%; a 80\%; a 70\%; água); coradas em hematoxilina de Harris por um minuto; desidratadas em alcoois crescentes (70\%; 80\%; absoluto duas vezes); clarificadas em xilol (duas vezes); montadas com resina acrílica e lamínula. O material fixado em formol a 10\% (mínimo de 5 horas) é incluído em parafina. Os blocos cortados e corados em hematoxilina/eosina são eventualmente submetidos às técnicas seletivas, de acordo com as necessidades de cada caso.

\section{RESULTADOS}

Os resultados revelaram a predominância dos gliomas (31,9\%), sendo o glioblastoma multiforme o mais frequente $(22,7 \%)$, seguido das patologias ligadas a AIDS, dentre elas o linfoma 
Tabela 1. Diagnóstico histológico dos 44 pacientes submetidos a biópsia estereotáxica.

\begin{tabular}{lcc}
\hline Etiologia & Número & $\%$ \\
\hline Glioblastoma multiforme & 10 & $22,7 \%$ \\
Linfoma primário do SNC & 8 & $18,2 \%$ \\
Encefalopatia pelo HIV & 4 & $9,1 \%$ \\
Leucoencefalopatia multifocal progressiva (LEMP) & 4 & $9,1 \%$ \\
Toxoplasmose & 4 & $9,1 \%$ \\
Metástase & 3 & $6,8 \%$ \\
Astrocitoma baixo grau & 3 & $6,8 \%$ \\
Vasculites & 2 & $4,6 \%$ \\
Astrocitoma anaplásico & 1 & $2,3 \%$ \\
Linfoma de células gigantes (diplóico) & 1 & $2,3 \%$ \\
Cisto aracnóideo & 1 & $2,3 \%$ \\
Tumor neuroectodérmico primitivo (PNET) & 1 & $2,3 \%$ \\
Doença de Creutzfeldt-Jakob & 1 & $2,3 \%$ \\
Nódulo fibroglial calcificado & 1 & $2,3 \%$ \\
Total & 44 & $100 \%$ \\
\hline
\end{tabular}

primário (18,2\%), leucoencefalopatia multifocal progressiva (LEMP: 9,1\%), leucoencefalopatia pelo HIV $(9,1 \%)$ e toxoplasmose $(9,1 \%)$. Três casos de metástases $(6,8 \%)$, um tumor neuroectodérmico primitivo (PNET: 2,3\%), um linfoma de células gigantes diplóico (2,3\%) e outras patologias não tumorais $(13,8 \%)$ (Tabela 1$)$.

A idade variou de 1 a 83 anos com a média de 47 anos. Até o momento tivemos mortalidade nula $(0 \%)$ relacionada ao procedimento. Nossa morbidade é de 2,3\%, decorrente de sangramento no leito tumoral em uma paciente de 76 anos com glioblastoma na região da pineal, prolongando sua internação hospitalar por 6 dias, tendo alta com a mesma sintomatologia prévia à BE (Fig 1).

Com relação a acurácia, nesse período o diagnóstico foi estabelecido em todos os casos. Em todos os procedimentos o neuropatologista esteve presente em sala.

Nosso paciente mais jovem foi um lactente de 1 ano, que tinha passado de retinoblastoma em ambos os olhos e que desenvolveu terceira lesão no III ventrículo. A BE revelou PNET, condição esta conhecida como "retinoblastoma trilateral"5.

Dez pacientes tiveram diagnóstico de glioblastoma multiforme. A idade variou de 40 a 83 anos e a localização predominante foi parietal, seguida por temporal, frontal, corpo caloso, talâmico e pineal. Um paciente jovem com astrocitoma anaplásico insular esquerdo, iniciou o quadro com crise convulsiva e alteração mental, sendo biopsiado prontamente e encaminhado para tratamento complementar. Três astrocitomas de baixo grau foram diagnosticados, o primeiro no terceiro ventrículo, outro na ínsula esquerda e outro no lobo parietal esquerdo. A idade média foi 30 anos.

Nos pacientes com AIDS, o linfoma primário foi o diagnóstico predominante, sendo 3 talâmicos, 1 frontal, 1 temporal e 1 parietal. Um paciente com lesão diplóica, mapeada por estereotaxia 


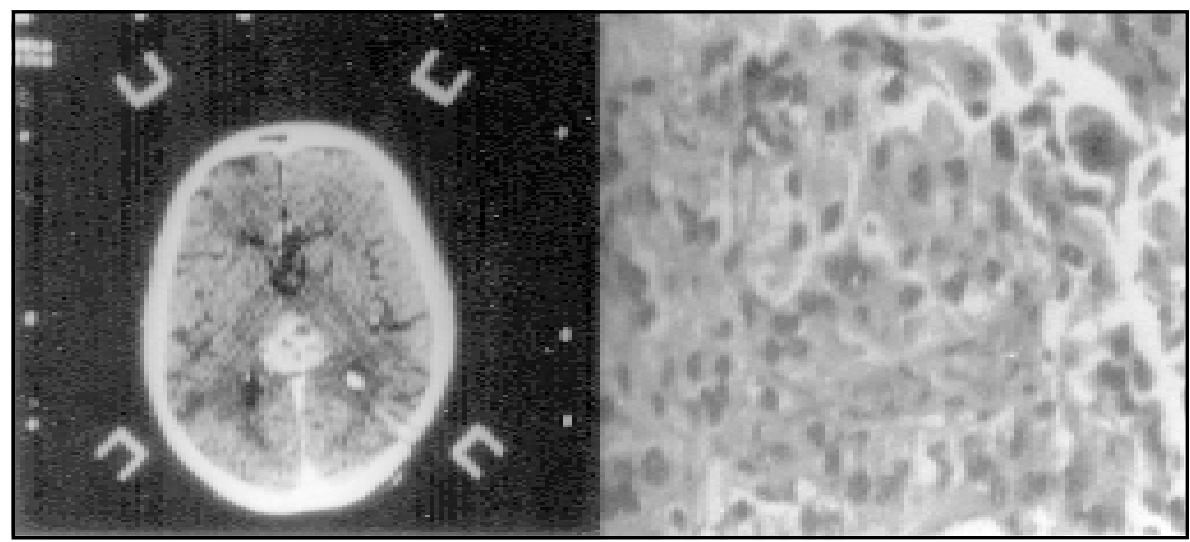

Fig 1. À esquerda, estereotomografia de crânio com contraste iodado mostrando lesão heterogênea, com intensa captação de contraste e pequenas áreas de necrose no seu interior, situada na região da pineal. À direita, a histologia da biópsia estereotáxica com os aspectos característicos de glioblastoma multiforme.

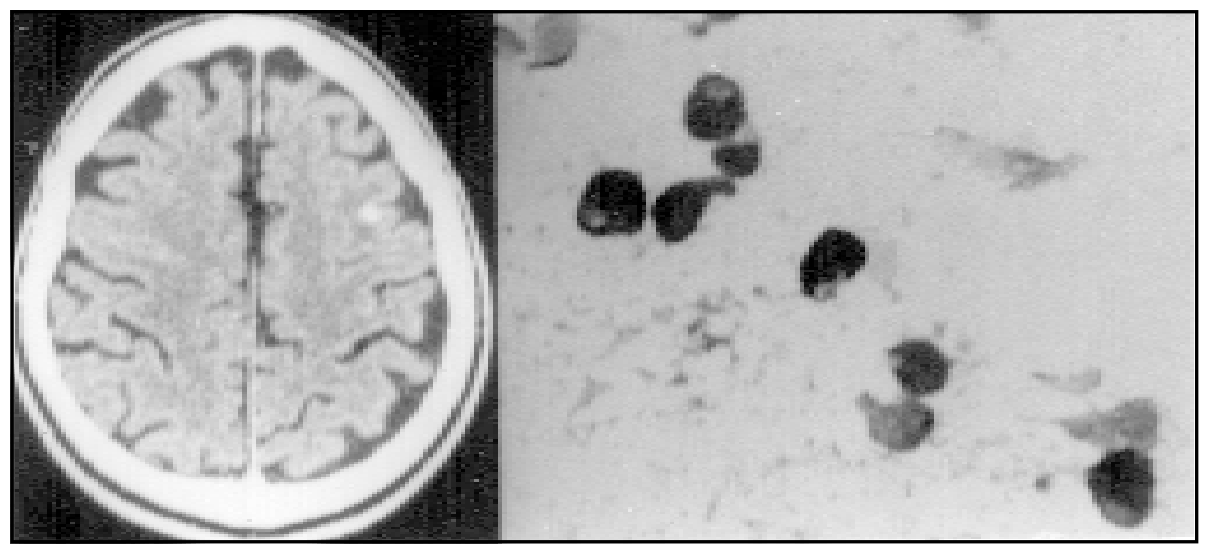

Fig 2. À esquerda, estereotomografia de crânio com contraste iodado mostrando lesão homogênea, com intensa captação de contraste, situada no lobo frontal esquerdo. À direita, a histologia da biópsia estereotáxica com presença de melanina, confirmando melanoma melanocítico metastático.

e biopsiada, teve diagnóstico de linfoma de células gigantes. Quatro casos de leucoencefalopatia multifocal progressiva (LEMP) foram diagnosticados sendo: 2 frontais e 2 na corona radiada fronto parietal. Outros 4 pacientes tiveram o diagnóstico de leucoencefalopatia pelo HIV, predominando na substância branca frontal, corona radiada e um no cerebelo. Quatro casos de toxoplasmose foram confirmados: frontal direito, núcleo lenticular esquerdo, III ventrículo e parietal esquerdo.

Um paciente idoso, HIV negativo, tinha duas lesões cerebrais periventriculares e uma cerebelar, que foi biopsiada, revelando-se linfoma primário.

Três casos de metástases múltiplas foram biopsiados (dois adenocarcinomas e um melanoma metastático não melanocítico - Fig 2). Em nenhum havia sido localizado o sítio primário.

Um caso de neuroinfecção, HIV negativo, com demência rapidamente progressiva, apresentava na RM do cérebro lesão de substância branca e intensa atrofia cerebral. Foi feita BE e biópsia cortical clássica guiada pela estereotaxia na área atrófica, que mostrou ser doença de CreutzfeldtJakob. 
Uma paciente, com história de hipertensão intracraniana e relato de dois episódios de crise convulsiva, mostrava na RM uma lesão hipointensa em T1 e hiperintensa em T2, sem captação de contraste, situada na região frontal. Foi sugerido na reunião de neuro-radiologia o diagnóstico de glioma de crescimento lento. Realizamos a $\mathrm{BE}$ da cápsula da lesão que revelou parede de cisto aracnóideo, tendo sido em seguida esvaziado o cisto por estereotaxia .

Dois casos de mulheres jovens, com história de abuso de drogas, apresentavam lesões na RM restritas aos giros no lobo temporal e parietal, em vigência de infecção, a primeira paciente com abscesso perianal e a segunda paciente com endocardite. A exploração angiográfica foi normal e a $\mathrm{BE}$ revelou vasculite. Os dois casos apresentavam em comum agravamento do quadro neurológico e progressão da lesão na RM e edema digitiforme na substânca branca, simulando lesão infecciosa ou tumoral. Uma paciente, com história de epilepsia há 15 anos, apresentou na investigação de rotina por TC uma lesão temporal medial hiperdensa com halo hipodenso. A RM confirmou os achados anteriores e o LCR foi normal. Foi biopsiada e revelou nódulo fibroglial calcificado.

\section{DISCUSSÃO}

O conceito de estereotaxia foi estabelecido por Clarke $(1906)^{6,7}$, em seu laboratório e aprovado por seu companheiro de instituição, Horsley que, a princípio, não acreditava em sua execução na prática clínica. Somente a partir de 1947, é que Spiegel iniciou a estereotaxia visando a psicocirurgia ${ }^{8}$ e logo em seguida Leksell (Estocolmo) e Talairach (Paris) ${ }^{4}$, também realizaram este procedimento, pois inicialmente havia pouco interesse com relação às neoplasias cerebrais. Com o advento da TC, uma revolução foi notada na apreciação, avaliação e evolução das patologias do sistema nervoso central (SNC) $)^{9,10,11}$. Com isto, as lesões cerebrais puderam ser diretamente acessadas e calculadas com a perfeita visão de seus limites. A TC acoplada a um sistema estereotáxico revela de maneira precisa e matemática a demonstração real e tridimensional de lesões do SNC. Recentemente pudemos lançar mão de estudos mais complexos que interagem TC, Angiografia e RM, graças ao desenvolvimento de sofisticados programas de informática que criam imagens interativas e orientam todo o trajeto do cirurgião, permitindo a preservação anatômica das estruturas neurovasculares importantes, delimitando toda a margem lesional. Esse sistema possibilita uma biópsia acurada e minuciosa ou uma ressecção cirúrgica aberta dentro dos seus limites reais ${ }^{4,12-15}$.

No planejamento da BE duas lesões merecem comentários. O primeiro, em relação as lesões vasculares que devem ter estudo de RM complementar, observando-se o fluxo intratumoral e, em muitos casos, afastando-se malformação artério-venosa e cavernomas. O segundo, em relação as lesões tumorais com grande efeito de massa e desvio importante da linha média; todos os cuidados devem ser tomados no sentido de evitar alguma complicação cirúrgica, que agravaria certamente o quadro do paciente. É importante salientar que a BCE prova ter alto valor diagnóstico. Morbidade e mortalidade são baixas, na nossa série foi de $2,3 \%$ e nas demais citadas na literatura está em torno de 2,5\% $\%^{2,4,14,16-18}$. Em $15 \%$ das biópsias, a maioria das grandes séries excluem tumor, sendo diagnosticada outra patologia ${ }^{18}$. Segundo Kelly ${ }^{4}$, não tem grande importância diferenciar um glioma alto grau e uma metástase, pois ambos iriam para a radioterapia. Contudo, o diagnóstico de abscesso, de aparência idêntica nas imagens, faria uma diferença significativa. O diagnóstico etiólogico foi obtido em todos estes pacientes. Isto se deve à presença do neuropatologista em sala, da atualização de técnicas em informática, do trabalho desenvolvido por um físico-médico e da discussão permanente entre os profissionais dos serviços de neuropatologia, neuro-radiologia e neurocirurgia.

No caso dos pacientes portadores de $\operatorname{AIDS}^{1,2,16,17,19,20}$, como ponto de partida é de fundamental importância o diagnóstico etiológico lançando-se mão do quadro clínico e exames complementares adequados (LCR, TC, RM). Contudo, em muitos casos se tornam insuficientes. Sabe-se que o sucesso terapêutico está intimamente relacionado à precisão no diagnóstico. A biópsia estereotáxica nas doenças infecciosas está indicado para as lesões pequenas, profundas e no tronco cerebral. Na série brasileira de Cescato /Teixeira ${ }^{16,17}$ em pacientes submetidos a biópsia estereotáxica, o resultado obtido 
no Hospital das Clínicas da Faculdade de Medicina da Universidade de São Paulo foi: mortalidade, $0 \%$; morbidade, $2,5 \%$; sucesso, $85 \%{ }^{17}$. As falhas foram atribuídas a escolha do alvo, profissionais inexperientes, dificuldade técnica na coleta, transporte ou armazenamento e falta de pessoal habilitado para processar material. A morbi-mortalidade é reduzida e o sucesso aumenta com o aprimoramento da técnica cirúrgica e histopatológica. A utilização de congelação ou técnicas de rotina no centro cirúrgico aumenta a margem de sucesso pela possibilidade de retirada de novas amostras no caso de normalidade ou inespecificidade do material biopsiado ${ }^{21}$.

Nossos resultados mostram os gliomas como o diagnóstico predominante, especialmente o glioblastoma multiforme e, por boa parte do nosso material incluir AIDS, o linfoma vem logo a seguir com destaque. Isto a nosso ver é a tendência de todos os grupos que, como o nosso, atendem a todo o tipo de patologia.

Concluindo, a BE é procedimento seguro e eficaz, desde que efetuado em condições ideais por profissionais habilitados, com instrumental adequado, associado a neuropatologista experiente com possibilidade de execução de técnicas histopatológicas ideais (microscopia óptica por congelação, rotina e técnicas especiais: imuno-histoquímicas e microscopia eletrônica). O insucesso está na falha da seleção do paciente, na escolha do alvo, na técnica neurocirúrgica ou na interpretação histológica.

\section{REFERÊNCIAS}

1. Levy RM, Pons VG, Rosemblum ML. Central Nervous mass lesions in the AIDS. J Neurosurg 1984;61:9-16.

2. Levy RM, Russel E. Yungbluth, Hidvegi DF, Brody BA, Canto MC. The efficacy of image guided stereotactic brain biopsy in neurologically symptomatic AIDS patients. Neurosurgery 1992;30:186-190.

3. Sedan R, Peragut JC; Vallicioni P. Presentation d'un appareillage original pour biopsie cérébrale et tumorale en conditions stéreotaxiques. Communication à la Société de Neurochirurgie de Langue Française, Dec 1975.

4. Kelly PJ. Tumor stereotaxis, Philadelphia: WB Saunders, 1991:1-220.

5. Bader JL, Meadows AT, Zimmerman LE, et al. Bilateral retinoblastoma: trilateral retinoblastoma. Cancer Genet Cytogenet 1982;5:201-213.

6. Clarke RH. Investigations of the central nervous system: methods and instruments. Jonhs Hopkins Hospital Report, Special Volume, 1920:1-162.

7. Horsley V, Clark RH. The structure and function of cerebellum examined by new method. Brain 1906;31:45-124.

8. Spiegel EA, Wycis HJ, Marks M, Lee A. Stereotactic apparatus for operations. Science 1947;106:439-450.

9. Apuzzo MLJ, Sabshin JK. Computed tomographic guidance stereotaxis in management of intracranial mass lesions. Neurosurgery 1983;12:277-285

10. Lobato RD, Rivas JJ, Cabello A. Stereotactic biopsy of brain lesions visualized with CT. Appl Neurophysiol 1982;45:426-430.

11. Schlitt MJ, Morawetz, Bonnin JM, Zeiger HE, Whitlety RJ. Brain biopsy for encephalitis. Clin Neurosurg 1986;33:591-602.

12. Kelly PJ. Volumetric stereotactic surgical ressection of intra-axial brain mass lesion. Mayo Clin Proc 1988;63:1186-1198.

13. Kondziolka D, Dempsey PK, Lunsford LD, Kestle JRW, Dolan EJ, Kanal L. A comparison between MRI and CT for stereotactic coordinate determination. Neurosurgery 1992;30:402-407.

14. Ostertag CB, Mennel HD, Kiessling M. Stereotactic biopsy of brain tumors. Surg Neurol 1980;14:275-283.

15. Thomas DGT, Kitchen ND. Stereotactic techniques for brain biopsies. Arch Dis Child 1993;69:621- 624.

16. Cescato VAS, Teixeira MJ. Biópsia Estereotáxica. In Nitrini R, Machado LR. Condutas em neurologia 1993. São Paulo: Clínica Neurológica HC/FMUSP, 1993:179-184.

17. Cescato VAS. Biópsia estereotáxica de lesões encefálicas inflamatórias In Machado LR, Nóbrega JPS, Livramento JP, Spina-França A. Neuroinfecção 94. São Paulo: Clínica Neurológica HC/FMUSP, 1994:249-252.

18. Friedman WA, Sceats DJ Jr, Nestok BR. The incidence of unexpected pathological findings in an image-guided biopsy series: a review of 100 consecutive cases. Neurosurgery 1989;25:180-184.

19. Chappel ET, Guthrie BL, Orestein J. The role of stereotactic biopsy in the management of HIV-related focal brain lesions. Neurosurgery 1992;30:825-829.

20. Prayson RA, Estes ML. Stereotactic brain biopsy for diagnosis of PML. South Mel J 1993;69:1381-1394.

21. Berkeley BB, Adams JH, Doyle D, Graham DI, Harper CG. The smear technique in the diagnosis of neurosurgical biopsies. N Z Med J 1978;87:12-15. 